\title{
Necesidades de los educadores para la atención socioeducativa del menor extranjero no acompañado en los centros de acogida
}

\author{
Alfonso Conde-Lacárcel ${ }^{1}$, Noelia Parejo-Jiménez ${ }^{1 *}$, Ernesto Maroto-Aguilera ${ }^{1}$ y María E. Parra-González ${ }^{2}$ \\ (1) Facultad de Ciencias de la Educación, Departamento de Métodos de Investigación y Diagnóstico en Educación \\ (MIDE), Universidad de Granada, Hospital Real, Cuesta del Hospicio s/n 18071 Granada, España. \\ (correo-e: alfconl8@ugr.es, noeliaparejo@ugr.es, ernestomaroto.jer@gmail.com) \\ (2) Facultad de Educación, Economía y Tecnología de Ceuta, Departamento de Métodos de Investigación y Diagnóstico \\ en Educación (MIDE), Universidad de Granada, Hospital Real, Cuesta del Hospicio s/n 18071 Granada, España. \\ (correo-e: elenaparra@ugr.es).
}

*Autor a quien debe ser dirigida la correspondencia.

Recibido Dic. 26, 2019; Aceptado Feb. 28, 2020; Versión final Abr. 27, 2020, Publicado Ago. 2020

\begin{abstract}
Resumen
El estudio que se presenta tiene como objetivo mostrar una descripción de la caracterización, formación e intervención llevada a cabo por educadores que atienden a menores extranjeros no acompañados en un centro de acogida de la ciudad de Granada, España. Para ello, hemos pasado un cuestionario a un grupo de diecinueve educadores cuyos resultados han sido analizados siguiendo una metodología cuantitativa. Los resultados obtenidos han puesto de manifiesto una serie de necesidades que presentan los educadores, relacionadas principalmente con estrategias específicas para llevar a cabo una intervención educativa con menores migrantes y la escasa coordinación y cooperación entre profesionales. Se concluye que es necesario atender a estas necesidades a través de un plan de formación que dote a los educadores de estrategias y herramientas para poder ofrecer una respuesta socioeducativa adecuada a los menores migrantes en los centros de acogida.
\end{abstract}

\section{Needs of educators for the socio-educational attention of unaccompanied foreign children in reception centers}

\begin{abstract}
The present study aims to show a description of the characterization, training, and intervention carried out by educators who attend unaccompanied foreign minors in a reception center in the city of Granada, Spain. Nineteen educators were surveyed with a questionnaire and that was then analyzed using a quantitative methodology. The results obtained revealed a series of needs presented by the educators surveyed. The main need was related to specific strategies to carry out an educational intervention with migrant minors and the poor coordination and cooperation among professionals. It is concluded that it is necessary to attend to these needs through a training plan that equips educators with strategies and tools to offer an adequate socioeducational response to migrant minors in reception centers.
\end{abstract}

Keywords: educator; inclusion; professional competence; unaccompanied foreign children 


\section{INTRODUCCIÓN}

El fenómeno migratorio no es algo nuevo ya que desde siempre ha existido y siempre relacionado con personas adultas. No obstante, en las últimas décadas se ha identificado la presencia de menores de edad en los procesos migratorios (Ortega, 2019). Concretamente en España, "este fenómeno comenzó a detectarse en 1996, inicialmente en las Islas Canarias, cuando se interceptó el primer barco con menores a bordo de África. Después del año 2000, hubo un auge en el número de inmigrantes menores sin familiares" (Salmerón - Manzano y Manzano - Agugliaro, 2018, p.2.). Así pues, a lo largo de los últimos años ha ido aumentado el número de menores migrantes no acompañados que llegan a España, especialmente a través de las ciudades autónomas de Ceuta, Melilla, las cuales han tenido que poner en marcha centros de recepción específicos para atender a este fenómeno migratorio (Martínez- Martínez, 2019). Ante esta situación, se hace necesario dar una respuesta legal. Para ello, a nivel internacional contamos con la Declaración Universal de los Derechos Humanos (1948); la Declaración de los Derechos del Niño (1959) y los acuerdos vinculantes establecidos en la Convención sobre los Derechos del Niño (1989). De esta última, destacamos el artículo 25 sobre la protección social de la infancia, el derecho a la educación; la importancia de la familia y derechos básicos de protección e identidad, respectivamente, entre otros muchos (Borrego, 2014).

A nivel Europeo, destaca la Carta Europea de los Derechos del Niño, la cual hace hincapié en garantizar la inclusión y el reconocimiento de los derechos y deberes fundamentales de los menores. $\mathrm{Y}$, a nivel nacional español encontramos algunas leyes como la de Protección Jurídica del Menor (1/1996 de 15 de Enero), de aplicación a los menores en situación de riesgo o desamparo tanto a nivel nacional como procedentes de otros países. Esta última, ha marcado puntos de inflexión en España con el fin de ofrecer la mejor intervención con los menores y la infancia desde las distintas administraciones a nivel nacional, autonómico o local. También, disponemos de la Ley Orgánica 8/2015, de 22 de julio, de modificación del sistema de protección a la infancia y a la adolescencia, la cual hace una revisión de todo el trabajo realizado hasta el momento actualizando aspectos relacionados con situaciones conflictivas en el seno familiar, problemas de comportamiento agresivo, serias dificultades para ejercer la responsabilidad de los padres, protección integral ante la violencia de género y menores migrantes.

Además de esto, es importante destacar que en otros países como por ejemplo en América Latina y Caribe, también está comenzando a tener importancia la migración de menores sin referentes familiares, ya que todos los años llegan niños/as sin compañía de adultos con una serie de necesidades específicas, las cuales requieren de atención. Ante esto, en los países de la región se han desarrollado una serie de avances para la protección de los derechos de los menores migrantes. De todos ellos, podemos destacar el desarrollo de una serie de protocolos y estándares para la protección y atención del menor migrante en la mayoría de los países de Centroamérica y el establecimiento de un espacio técnico de colaboración para tratar los temas específicos de los menores migrantes en la Conferencia Regional sobre Migración (CEPAL y UNICEF, 2018).

De una forma u otra, aunque con algunas controversias, estos textos legales velan por el cumplimiento del derecho fundamental a la educación de todas las personas. Es indiscutible, que "la educación representa un proceso por medio del cual el ser humano adquiere distintas herramientas para su inserción en la sociedad y su realización personal" (Castillo y Gamboa, 2012, p.56), siempre y cuando se lleve a cabo de una forma adecuada. Así pues, cuando esta escolaridad o la continuidad en el sistema educativo no está asegurada, se niega la inclusión y un futuro laboral al menor (Expósito et al., 2019). Para estos menores sin referentes familiares tanto la figura del maestro o profesor en los centros educativos como la del educador en los centros de acogida, se convierte en indispensable para alcanzar dicho propósito. Estos profesionales, tienen en común promover un desarrollo integral del menor migrante en los ámbitos académico, social y laboral que le permita obtener un empleo, siendo éste considerado como "la mejor protección contra la exclusión social" por la Unión Europea (Fernández, 2010).

En la actual sociedad de la información y del conocimiento, la globalización, el desarrollo de las tecnologías de la información y la comunicación (TIC, en adelante), el aumento de la migración, etc. están produciendo cambios en la forma de trabajar de estos educadores (Pedrazza et al., 2018), los cuales deben desarrollar una serie de competencias para dar respuesta a las nuevas necesidades que se les presentan. Una de estas necesidades, es la de adaptarse a las características, intereses y motivaciones individuales de cada uno de estos menores, por lo cual se requiere a educadores que sean capaces de implementar una educación adaptativa (Jason y Westberg, 2018). Por aprendizaje adaptativo, se entiende dar a cada uno lo que necesita en función de sus conocimientos, habilidades para el aprendizaje, motivaciones, etc.; $y$ esto es posible gracias a las TIC. Existen plataformas tecnológicas que son capaces de ofrecer un aprendizaje adaptado a las necesidades del menor ya que disponen de múltiples rutas de aprendizaje, evaluación continuada, redirección del conocimiento y habilidades necesarias, modalidades de instrucción personalizadas, etc. (Dziuban et al., 2018). También, "este tipo de tecnologías favorece la adquisición del conocimiento por parte de los 
estudiantes y brinda mecanismos que despiertan el interés de éstos, proporcionando un ambiente de aprendizaje continuo y ubicuo, es decir, accesible en cualquier momento y lugar" (Ovalle et al, 2014, p.141).

La creación de este tipo de espacios de aprendizaje, permitiría que se llevase a cabo un trabajo coordinado entre los educadores de los centros de acogida y los docentes de los centros educativos donde los menores se encuentran escolarizados. El trabajo colaborativo entre los distintos profesionales es un factor clave para brindar una atención educativa de calidad e integral que ofrezca una respuesta coherente a las necesidades de una determinada población. Ésta se basa en el trabajo conjunto y una conciencia de colaboración interprofesional (Tsakitzidis et al., 2015) que permite que todos los profesionales que trabajan con menores migrantes partan de sus intereses y características de una forma más precisa, y fomenten una intervención que vaya en una misma línea de acción, de forma que sea lo más integral posible y sin contradicciones.

En este ambiente de aprendizaje adaptativo y de colaboración, los educadores de los centros de acogida deben desempeñar una serie de funciones. Es importante destacar, que apenas existe literatura específica sobre el trabajo que desempeñan los educadores con los menores migrantes no acompañados. A esto hay que sumar, que los perfiles profesionales de las personas que trabajan en los centros de acogida son distintos, pudiéndonos encontrar trabajadores sociales, educadores sociales, maestros de diferentes especialidades, etc. De ahí que en este artículo utilicemos el término general de "educadores". Ante esta situación, se hace difícil establecer qué funciones deberían tener estos profesionales. Desde una perspectiva general, diremos que estos profesionales deben tener competencias, las cuales están formadas por tres dimensiones que son: el saber, el saber hacer y el saber estar. Como se trata de trabajar con menores migrantes, a esta competencia habría que añadir la vertientes intercultural, por tanto los educadores que trabajen con menores migrantes deberían tener "competencias interculturales" (Jiménez, 2011).

Basándonos en la noción de "competencia intercultural" así en las distintas competencias recogidas el Real Decreto 1393/2007 por el que se establece la ordenación de las enseñanzas universitarias y en el Marco Español de Cualificaciones para la Educación Superior (MECES) para la formación de los distintos profesionales que son susceptibles de trabajan en los centros de acogida, y centrándonos en el perfil del educador, desde nuestro punto de vista y de una forma general, podemos decir que los educadores deberían: poseer una serie de conocimientos generales para atender a los menores migrantes (principios psicopedagógicos, sociológicos, normativos, estructura de los sistemas educativos y socio- comunitarios, etc.); realización de diagnósticos personales teniendo en cuenta los distintos contextos y situaciones personales; intervención educativa: diseño, implementación y evaluación de programas o acciones socioeducativas que incluyan contenidos sobre el desarrollo personal, educativo y social; y actitudes (crítica, analítica, aprecio por la diversidad, fomento de la igualdad, cooperación, flexibilidad, comprensión, diálogo, compromiso ético, motivación, etc.).

Actualmente, los menores migrantes no están motivados por su aprendizaje, manifestándose en algunas ocasiones el absentismo en estos niños e incluso abandono definitivo del sistema educativo y centros de acogida del país receptor. Este absentismo escolar indica que el sistema educativo no está atendiendo eficazmente la necesidades de estos menores y deberíamos preguntarnos que se está haciendo mal para alejar a los estudiantes de las escuelas (Shute y Cooper, 2015). Por este motivo, este estudio tiene como principal objetivo conocer cómo trabajan y qué necesidades tienen los educadores y educadoras a la hora de atender a los menores migrantes en los centros de acogida.

Atendiendo a la Agenda 2030 para el Desarrollo Sostenible, en la cual se reivindica la igualdad de género como esencial para el cumplimiento de todos los objetivos que en ella se exponen, incluido los derechos de todos los niños, niñas y adolescentes (CEPAL y UNICEF, 2018), en este artículo se analizan cuáles son las necesidades tanto de los educadores como de las educadoras que trabajan en los centros de acogida con el objetivo de poder dar una respuesta futura a todas ellas promoviendo la equidad y la justicia social de todas las personas implicadas en estos procesos de enseñanza - aprendizaje.

\section{MÉTODO}

En este estudio se pretende caracterizar la forma de trabajar con menores migrantes sin referentes familiares de un grupo 19 educadores de un centro de acogida de la ciudad de Granada, España. La muestra ha sido seleccionada de forma intencional siguiendo los criterios de conocimiento del centro, permiso de acceso, y trabajadores en activo que atiendan socioeducativamente a menores migrantes sin referente familiar en su puesto laboral. En cuanto a las características de la muestra, decir que está formada por un 68,4\% de mujeres y $31.6 \%$ hombres, con una predominancia de edad de entre 36 y 40 años $(42,1 \%)$, de los cuales $31,6 \%$ son solteros/as, el $26,3 \%$ casados/as y el $21,1 \%$ de divorciados/as. Además de esto, es importante destacar que el $52,6 \%$ tienen una formación universitaria de grado y el $47,4 \%$ de posgrado $(31,6 \%$ Máster y $15,8 \%$ Doctorado). 
La metodología empleada en este estudio es de corte cuantitativo. Hemos partido del cuestionario creado por Torres - Martín y Maroto (2016) ya validado a nivel empírico (Alfa de Cronbrach de ,948 para un intervalo de confianza del 95\%), redefiniendo, ajustando, e integrando nuevas cuestiones en base a las indicaciones de los expertos seleccionados para la validación de contenido por jueces expertos a través de la técnica Delphi. Esta estrategia persigue a través de un proceso reiterado, secuencial y realizado por un grupo de expertos participantes, validar o refutar un posible instrumento de investigación (en este caso el cuestionario empleado) o dar respuesta consensuada a una problemática planteada, entre otros aspectos, buscando la convergencia y estabilidad de las respuestas dadas por los participantes.

De acuerdo con Cabero e Infante (2014): "el método Delphi es posiblemente uno de los más utilizados en los últimos tiempos por los investigadores para diferentes situaciones y problemáticas, que van desde la identificación de tópicos a investigar, especificar las preguntas de investigación, identificar una perspectiva teórica para la fundamentación de la investigación, seleccionar las variables de interés, identificar las relaciones causales entre factores, definir y validar los constructos, elaborar los instrumentos de análisis 0 recogida de información, o crear un lenguaje común para la discusión y gestión del conocimiento en un área científica" (p.2). Finalmente, el instrumento utilizado está compuesto por 64 ítems con opciones de respuesta dicotómicas, de escala y abiertas. Estos ítems se dividen en cuatro dimensiones que son: caracterización de los educadores, formación del educador, intervención con los menores migrantes, grado de satisfacción con el puesto de trabajo y sugerencias de mejora.

Para el análisis de los datos hemos realizado un análisis descriptivo e inferencial empleado el programa estadístico SPSS v.22. En lo que respecta al análisis descriptivo, se ha analizado la frecuencia de respuesta, la media y la desviación típica para conocer las necesidades generales de los educadores de este centro. A nivel inferencial, se ha aplicado la $U$ de Mann - Whitney, con el objetivo de identificar si existen diferencias significativas entre las necesidades o metodología de trabajo de hombres y mujeres, para poder dar una respuesta más adecuada a la misma en futuros programas de formación. Se ha aplicado este estadístico, debido a que tras realizar la prueba de Shapiro - Wilk y la prueba de Levene no podemos afirmar con total seguridad que la muestra tenga una distribución normal. Además de esto, es más adecuada utilizar la la $U$ de Mann - Whitney cuando se trata de muestras pequeñas como la de este estudio. El valor de significación que se ha tenido en cuenta en la realización de estos estadísticos inferenciales es $p=.05$.

\section{RESULTADOS Y DISCUSIÓN}

En este apartado, se presentan los resultados obtenidos en la investigación, los cuales nos permiten caracterizar el trabajo que llevan a cabo los educadores que trabajan con menores migrantes no acompañados en el centro de acogida y describir qué necesidades tienen.

\section{Formación del educador}

En primer lugar, encontramos diferencias significativas en la titulación académica entre hombres y mujeres: en mayor medida los hombres poseen estudios de posgrado frente a las mujeres que poseen principalmente estudios de grado $(U=10.000$ y $p=.008)$. Además de esto, es importante destacar que el ámbito de trabajo previo al actual es desarrollado en mayor medida en educación no formal por parte de las educadoras y en educación de adultos por parte de los educadores. En concreto, a esta segunda cuestión un 52,6\% no respondieron qué ámbito laboral habían ejercido previamente, y de los que respondieron tenemos a un $26,3 \%$ que han trabajado en educación no formal, un10,5\% en educación de adultos, un 5,3\% en educación secundaria y un 5,3\% en formación profesional.

En segundo lugar, en cuanto a los años de experiencia previa en relación al trabajo actual, un $26,3 \%$ afirma tener entre 0-5 años de experiencia y un $21,1 \%$ una experiencia previa en el puesto de 6-8 años. Al mismo tiempo, la estabilidad en el puesto de trabajo parece ser lo suficiente larga como para desarrollar los programas e intervenciones planificadas (36,8\% de 0-3 años; $26,3 \%$ de $8-11$ años y $15,8 \%$ entre $4-7$ años y de 12-15 años). En lo que respecta a su situación contractual actual, creemos que es adecuada para una correcta intervención con los menores puesto que es fija-indefinida (52,6\%) o de prioritaria-definitiva $(26,3 \%)$. En sus puestos, mayoritariamente cumplen funciones de educadores de apoyo $(42,1 \%)$, aunque otros son componentes del equipo técnico o educadores responsables $(21,1 \%)$.

A nivel de formación continua para intervenir con los menores migrantes, centrándonos en el conjunto de los datos (educadores y educadoras), nos encontramos con un $84,2 \%$ que dice no haber asistido a cursos formativos sobre intervención con menores en riesgo de exclusión social; apenas un 10,5\% ha recibido formación al respecto. Igualmente es significativo el desconocimiento de la metodología específica del centro sobre la atención a estos menores en un $57,9 \%$, habiendo encontrado una disparidad entre aquellos 
educadores que no han recibido cursos de formación en metodologías de intervención y los que sí (42,1\% frente al $57,9 \%$ respectivamente). La formación previa a la entrada a los centros fue realizada en su mayoría durante la carrera $(68,4 \%)$ y en menor medida a lo largo de la carrera profesional $(15,8 \%)$. Es importante destacar que han recibido formación previa durante la carrera sobre atención a la diversidad $(84,2 \%)$ siendo escaso el porcentaje de educadores que realizaron la formación previa a lo largo de la carrera profesional (10,5\%). En cuanto a la formación en el área para promover el desarrollo socio-personal de estos menores, decir que un $57,9 \%$ afirma haberla recibido durante el periodo de contratación.

Además de todo esto, es importante destacar que en lo que respecta a esta formación continua existen diferencias significativas entre los educadores y las educadoras. Los hombres han asistido en mayor medida a cursos sobre metodología con menores migrantes $(U=11.500$ y $p=.009)$ y en atención a la diversidad $(U$ $=19.500$ y $p=.007)$. La formación de éstos ha sido principalmente previa a la entrada en los centros. También, existen diferencias significativas entre el conocimiento que tienen los educadores y las educadoras sobre la metodología que lleva a cabo el centro para atender a menores $(U=15.000$ y $p=.014)$, teniendo un mayor conocimiento los hombres sobre este aspecto.

Estos datos son un claro reflejo de cómo actualmente el tema de la formación de los educadores que trabajan con la niñez migrante sin referentes familiares ha sido poco estudiado y cómo las ofertas formativas para los profesionales que atienden a estos menores en riesgo de exclusión social son escasas y se encuentran poco fundamentadas en la investigación. En cierto modo, esto lo pone de manifiesto Salmerón- Manzano y Manzano - Agugliaro, 2018) en su estudio bibliométrico realizado sobre las principales tendencias de investigación a nivel mundial en torno a los menores no acompañados. Este análisis describe cómo en el campo de las ciencias sociales predominan los estudios relacionados con implicaciones legales y políticas de los menores no acompañados. Así pues, este mismo autor afirma que desde un punto de vista social hay pocos estudios sobre modelos de intervención para atender a las necesidades de estos menores en términos de educación, de ahí radica la importancia de investigaciones de este tipo.

A continuación, a modo ilustrativo, se muestra la Tabla 1 en la que se exponen las estadísticas de grupo obtenidas con respecto a la formación del educador de algunos de los ítems que se han ido comentado anteriormente. En ella se pueden apreciar en mayor detalle las medias de las respuestas dadas por los educadores y educadoras a cada uno de los ítems, y las diferencias entre ellas. Para la interpretación dela tabla, es importante tener en cuenta que se ha seguido en las respuestas a los ítems una escala tipo Likert: 1 = "nunca", 2 = "pocas veces", $3=$ "a veces" y 4 = "siempre".

Tabla 1: Estadísticas de grupo. Formación del educador

\begin{tabular}{|c|c|c|c|c|c|}
\hline \multicolumn{2}{|l|}{ Variable: Sexo } & \multirow[t]{2}{*}{$N$} & \multirow{2}{*}{$\begin{array}{l}\text { Media } \\
2.3333 \\
\end{array}$} & \multirow{2}{*}{$\begin{array}{l}\begin{array}{l}\text { Desviación } \\
\text { estándar }\end{array} \\
2.06559 \\
\end{array}$} & \multirow{2}{*}{$\begin{array}{l}\text { Media de error } \\
\text { estándar } \\
.84327\end{array}$} \\
\hline Asistencia a c & Hombre & & & & \\
\hline & Mujer & 13 & 1.0769 & .27735 & .07692 \\
\hline \multirow{2}{*}{$\begin{array}{l}\text { Asistencia a cursos formativos relacionados con la metodología } \\
\text { utilizada en la atención de menores extranjeros no acompañados }\end{array}$} & Hombre & 6 & 4.6667 & .81650 & .33333 \\
\hline & Mujer & 13 & 2.2308 & 1.78 & 54 \\
\hline \multirow{2}{*}{$\begin{array}{l}\text { Conocimiento por parte del educador/a de la metodología que } \\
\text { utiliza el centro para la atención a menores migrantes no } \\
\text { acompañados }\end{array}$} & Hombre & 6 & 2.0000 & .00000 & .00000 \\
\hline & Mujer & 13 & 1.3846 & .50637 & .14044 \\
\hline \multirow{2}{*}{$\begin{array}{l}\text { Formación previa a la entrada al centro en atención con menores } \\
\text { migrantes }\end{array}$} & Hombre & 6 & 4.0000 & 2.19089 & .89443 \\
\hline & Mujer & 13 & 2.1538 & .68874 & .19102 \\
\hline \multirow{2}{*}{$\begin{array}{l}\text { Formación previa a la entrada al centro en atención a la } \\
\text { diversidad }\end{array}$} & Hombre & 6 & 3.6667 & 1.96 & .80277 \\
\hline & Mujer & 13 & 2.0000 & .00000 & .00000 \\
\hline $\begin{array}{l}\text { Formación en el área de desarrollo socio-personal durante el } \\
\text { periodo de contratación }\end{array}$ & $\begin{array}{l}\text { Hombre } \\
\text { Mujer }\end{array}$ & $\begin{array}{ll}6 \\
13\end{array}$ & $\begin{array}{l}1.3333 \\
1.6923\end{array}$ & $\begin{array}{l}.51640 \\
.48038 \\
\end{array}$ & $\begin{array}{l}.21082 \\
.13323 \\
\end{array}$ \\
\hline $\begin{array}{l}\text { Formación previa recibida en el área de desarrollo socio - } \\
\text { personal }\end{array}$ & $\begin{array}{l}\text { Hombre } \\
\text { Mujer }\end{array}$ & $\begin{array}{ll}6 \\
13\end{array}$ & $\begin{array}{l}1.1667 \\
1.1538\end{array}$ & $\begin{array}{l}.40825 \\
.37553\end{array}$ & $\begin{array}{l}.16667 \\
.10415\end{array}$ \\
\hline
\end{tabular}

\section{Intervención con los menores migrantes}

A nivel general, el 73,7\% acumulado de los educadores afirma no participar "siempre" en el diseño de las acciones educativas que se realizan con los menores migrantes; y llevar a cabo una escasa planificación de la acción tutorial con el fin de dotarla de contenidos (47,4\% "a veces", $26,3 \%$ "siempre"). No obstante, sí participan en mayor medida en la planificación de acciones tutoriales de los menores en los dispositivos de atención (un 47,4\% "a veces" frente a un 26,3\% que lo hace "siempre"; un 21,1\% que lo hace "pocas veces" y un 5,3\% que dice no realizarlo "nunca"). Esto es contrario a lo que es definido por el análisis realizado por Martínez et al. (2016) en el que se expone la necesidad de una planificación de la Acción Tutorial para que sea un factor clave en la calidad de la formación integral de las personas. 
En esta misma línea, en aspectos referidos a la intervención directa de los educadores sobre las dificultades de aprendizaje de los menores en los centros de acogida, destacar un $36,8 \%$ que realiza acciones "siempre" frente al resto que lo hace de manera esporádica. Además de esto, el $42,1 \%$ de los encuestados manifiestan realizar "siempre" talleres para favorecer la inclusión social de los menores y un 59,9\% dicen realizarlos conjuntamente con agentes externos de manera esporádica. La principal colaboración que se establece entre los profesionales que intervienen con estos menores se lleva a cabo entre educadores de distintos ámbitos profesionales a la hora de la realización de la programación (31,6\% "siempre", 31,6\% "a veces" 31,6\% "pocas veces" y 5,3\% "nunca"), en contraposición a la falta de colaboración o apoyo con el equipo técnico dentro de los centros.

Así pues, en relación al trabajo cooperativo entre el equipo técnico y los educadores para desarrollar el proceso de intervención educativa con menores migrantes, se presenta también como variable a mejorar produciéndose un 5,3\% "nunca", un 31,6\% "pocas veces", un 47,4\% "a veces" y un $15,8 \%$ de manera continuada. Esto se pone de manifiesto también en la coordinación y asesoría por parte del equipo técnico en materia de acción tutorial que reciben los educadores, un $42,1 \%$ de los encuestados afirma recibirla "a veces", un $21,1 \%$ "siempre", un $26.3 \%$ "pocas veces" y un 10,3\% "nunca". Además de esto, el asesoramiento por parte del equipo técnico sobre atención a la diversidad es esporádico $(78,9 \%$ acumulado rangos "nunca - a veces"); y la facilitación de material para menores con necesidades específicas de apoyo educativo que facilita el equipo técnico a los educadores se produce un $36,8 \%$ "a veces", un $26,3 \%$ "siempre" y un $21,1 \%$ "pocas veces". En este punto, es importante destacar que la respuesta dada sobre la participación en la elaboración de las acciones educativas, y la coordinación o asesoría de la acción tutorial por parte del equipo técnico es más positiva por parte de los educadores.

Esta necesidad de colaboración entre los profesionales de los centros de acogida, también se ha puesto de manifiesto en otros estudios como el desarrollado por Mikkonen et al. (2019) sobre las percepciones que tienen los educadores acerca de su competencia en educación. Este estudio expone que los propios profesionales reconocen la importancia de llevar a cabo una colaboración entre ellos para la mejora del desempeño de su trabajo. A toda esta falta de colaboración, hay que añadir que solo el $26,3 \%$ de la muestra afirma recibir materiales e instrumentos por parte del centro "siempre", frente al $36,8 \%$ que afirma recibirlos "pocas veces" o "a veces". Estos resultados son un reflejo de lo que UNICEF (2019) pone de manifiesto en uno de sus últimos informes en el cual lleva a cabo una evaluación general de la situación de los menores migrantes que llegan a España. En este informe se expone, entre otros muchos aspectos, los déficits e inadaptación de las respuestas dadas a estos niños debido, entre otros factores, a la falta de recursos humanos y materiales disponibles en las diferentes instituciones estatales y autonómicas.

En lo referente a los contenidos trabajados en las intervenciones con menores migrantes, en relación a los valores culturales del país de acogida incluidos en las programaciones socioeducativas, podemos comprobar que el $42,1 \%$ de los educadores/as de los centros dice trabajarlo con los menores "a veces", seguido de un $31,6 \%$ que manifiesta realizarlo "pocas veces" y solamente un $26,3 \%$ "siempre". En cuanto a la atención a la cultura de procedencia del menor en el desarrollo de las intervenciones el $31,6 \%$ dice incluirlo "pocas veces" en la programación socioeducativa, seguido de $36.8 \%$ que dice hacerlo "a veces" y un $31,6 \%$ que lo hace siempre; los derechos y deberes de los menores en la elaboración de las programaciones son tenidos en cuenta "pocas veces" en un $36,8 \%$, "a veces" en un $26,3 \%$ y "siempre" en un $36,8 \%$; y la participación ciudadana es tenida en cuenta en las planificaciones un $42,1 \%$ " pocas veces", $21,1 \%$ a veces y un $36,8 \%$ "siempre".

En cuanto, a los objetivos y contenidos en relación a la autoestima, el autoconcepto, la inteligencia emocional, las habilidades sociales, el pensamiento crítico y creativo, la autonomía personal y la cotidianeidad y el establecimiento de rutinas, $84,2 \%$ de los encuestados manifiestan tenerlos en cuenta frente a un $15,8 \%$ de ellos que dicen no hacerlo. En esta línea, el estudio realizado por Rodorigo et al. (2019), ha puesto de manifiesto la falta de conocimiento por parte de los profesores en la mediación cultural; la conceptualización de ésta como algo puntual; la inexistencia de trabajo colaborativo entre los profesionales y la gran influencia que tiene la estructura organizativa y cultural de los centros educativos en la labor de profesionales externos, lo cual hace que su labor se desvirtúe. Por tanto, si desde los centros y las escuelas no se trabaja la interculturalidad es imposible que se consiga una plena inclusión de los menores no acompañados en la sociedad. Por otra parte, los educadores afirman que los menores tienen conocimiento de su objetivo final de conseguir la autonomía personal (52,6\% "siempre" frente a un 47,4\% "a veces) aunque la dinámica principal de trabajo provoque que "pocas veces" se establezcan reuniones periódicas entre el educador y el menor $(47,4 \%)$; solo un $26,2 \%$ dice realizarlas "siempre"; de todas formas, a lo largo de todo el proceso formativo se les proporciona información, material e instrumentos que puedan precisar (52,6\% "siempre" frente a un $26,3 \%$ "pocas veces" y un $21,1 \%$ "a veces"). Adquieren valores similares la presentación de los contenidos a los menores y las actitudes abiertas al diálogo por parte de los educadores/as que son llevados a cabo "a veces" en un $52,6 \%$ y "siempre" en un $26,3 \%$ durante las acciones formativas. No obstante, nos resulta bastante 
Ilamativo los bajos niveles obtenidos en el ítem referido a la existencia de consenso entre lo que necesita el menor y lo que quiere hacer el educador, es decir en la planificación del itinerario socioeducativo, ya que la mayoría de los educadores contestan que lo llevan a cabo "a veces" o "pocas veces".

Por último, en cuanto a la evaluación conjunta del itinerario formativo entre educador y el menor; la discusión de los resultados con otros profesionales; y la realización de evaluaciones psicopedagógicas previas para atender a la diversidad han obtenido resultados similares: un 26,3\% manifiesta llevarlo a cabo "siempre" frente a un $42,1 \%$ que lo hace "a veces" y un $26,3 \%$ que manifiesta hacerlo "pocas veces". La elaboración de informes psicopedagógicos finales sobre los menores por parte de los educadores fluctúa entre un $47,4 \%$ que dice realizarlos "siempre" y un $31,6 \%$ y $21,1 \%$ que los realizan "a veces" o "pocas veces" respectivamente. Ya antes mencionábamos la importancia que tiene el trabajar en equipo para los educadores y por tanto también en le evaluación. Además de esto, es importante mencionar que trabajar en colaboración con los menores migrantes e implicarlos en actividades como su propia evaluación también tiene ventajas para su formación. Es más, existen investigaciones como la realizada por Martos - García et al. (2017) que pone de manifiesto la satisfacción de los estudiantes con una evaluación formativa y colaborativa reconociendo que tiene beneficios y aspectos positivos en el proceso de enseñanza - aprendizaje como por ejemplo la participación activa, el aprendizaje colaborativo y la interrelación entre la teoría y la práctica.

A continuación, a modo ilustrativo, se muestra la Tabla 2 en la que se exponen las estadísticas de grupo obtenidas en algunos ítems referidos a la intervención que realiza el educador con menores migrantes. En ella se pueden apreciar en mayor detalle las medias de las respuestas dadas por los educadores y educadoras a cada uno de los ítems, y las diferencias entre ellas. Para la interpretación de la tabla, es importante tener en cuenta que se ha seguido en las respuestas a los ítems una escala tipo Likert: 1 = "nunca", 2 = "pocas veces", $3=$ "a veces" y 4 = "siempre".

Tabla 2: Estadísticas de grupo. Intervención con los menores migrantes

\begin{tabular}{|c|c|c|c|c|c|}
\hline \multicolumn{2}{|l|}{ Variable 1: Sexo } & \multirow{2}{*}{\begin{tabular}{l|l|l}
$N$ \\
6
\end{tabular}} & \multirow{2}{*}{\begin{tabular}{|l|} 
Media \\
1.6667 \\
\end{tabular}} & \multirow{2}{*}{$\begin{array}{l}\begin{array}{l}\text { Desviación } \\
\text { estándar }\end{array} \\
.51640\end{array}$} & \multirow{2}{*}{\begin{tabular}{|l|}
$\begin{array}{l}\text { Media de error } \\
\text { estándar }\end{array}$ \\
.21082
\end{tabular}} \\
\hline Conocimiento del plan de intervención educativa con los menores del & Hombre & & & & \\
\hline centro & Mujer & 13 & 1.6154 & .50637 & .14044 \\
\hline \multirow{2}{*}{$\begin{array}{l}\text { Elaboras o participas en el diseño de las acciones educativas con los } \\
\text { menores migrantes }\end{array}$} & Hombre & 6 & 3.1667 & .98319 & .40139 \\
\hline & Mujer & 13 & 2.7692 & .72501 & 20108 \\
\hline $\begin{array}{l}\text { Coordinación o asesoría de la acción tutorial por parte del equipo } \\
\text { técnico }\end{array}$ & \begin{tabular}{|l|}
$\begin{array}{l}\text { Hombre } \\
\text { Mujer }\end{array}$ \\
\end{tabular} & $\begin{array}{l}6 \\
13\end{array}$ & \begin{tabular}{|l|}
3.0000 \\
2.6154 \\
\end{tabular} & $\begin{array}{l}1.26491 \\
.76795 \\
\end{array}$ & $\begin{array}{l}.51640 \\
.21299 \\
\end{array}$ \\
\hline \multirow[t]{2}{*}{ Se lleva a cabo una acción tutorial en tu dispositivo de atención } & Hombre & 6 & 3.5000 & .83666 & .34157 \\
\hline & Mujer & 13 & 3.0000 & .70711 & .19612 \\
\hline \multirow[t]{2}{*}{ Planificación de la acción tutorial para dotarla de contenidos } & Hombre & 6 & 3.3333 & .81650 & .33333 \\
\hline & Mujer & 13 & 2.7692 & .83 & .23077 \\
\hline \multirow{2}{*}{$\begin{array}{l}\text { El centro se proporciona materiales e instrumentos para la acción } \\
\text { educativa a los educadores }\end{array}$} & Hombre & 6 & 3.1667 & .98319 & .40139 \\
\hline & Mujer & 13 & 2.7692 & .72501 & .20108 \\
\hline \multirow{2}{*}{$\begin{array}{l}\text { Se facilita asesoramiento por el equipo técnico sobre competencias } \\
\text { para la atención a la diversidad }\end{array}$} & Hombre & 6 & 3.1667 & .98319 & .40139 \\
\hline & Mujer & 13 & 2.5385 & .87706 & .24325 \\
\hline \multirow{2}{*}{$\begin{array}{l}\text { El equipo técnico facilita instrumentos o materiales para sujetos con } \\
\text { necesidades específicas de apoyo educativo }\end{array}$} & Hombre & \begin{tabular}{l|l}
6 & \\
\end{tabular} & 3.0000 & 1.26491 & .51640 \\
\hline & Mujer & 13 & 2.6154 & .96077 & .26647 \\
\hline \multirow[t]{2}{*}{ Realización de talleres para la inclusión social de los menores } & Hombre & 6 & 3.5000 & .83666 & .34157 \\
\hline & Mujer & 13 & 3.0000 & .81650 & .22646 \\
\hline \multirow{2}{*}{$\begin{array}{l}\text { Reuniones entre educador y el menor para planificar el itinerario } \\
\text { socioeducativo }\end{array}$} & Hombre & 6 & 3.0000 & .89 & ,36515 \\
\hline & Mujer & 13 & 2.5385 & .77625 & .21529 \\
\hline \multirow[t]{2}{*}{ Presentación de los contenidos al menor } & Hombre & 6 & 3.5000 & .83666 & .34157 \\
\hline & Mujer & 13 & 2.9231 & .64051 & .17765 \\
\hline \multirow[t]{2}{*}{ Actitud abierta al dialogo y cambios durante las acciones educativas } & Hombre & 6 & 3.3333 & .81650 & .33333 \\
\hline & Mujer & 13 & 2.9231 & .64051 & .17765 \\
\hline \multirow{2}{*}{$\begin{array}{l}\text { Se presta atención a la cultura de procedencia del menor en la } \\
\text { elaboración de objetivos y contenidos }\end{array}$} & Hombre & 6 & 3.5000 & .83666 & .34157 \\
\hline & Mujer & 13 & 2.7692 & .72501 & .20108 \\
\hline \multirow{2}{*}{$\begin{array}{l}\text { Grado de incorporación de los contenidos culturales del país de } \\
\text { acogida en los objetivos y contenidos educativos }\end{array}$} & Hombre & 6 & 3.1667 & .75277 & .30732 \\
\hline & Mujer & 13 & 2.8462 & .80064 & .22206 \\
\hline \multirow{2}{*}{$\begin{array}{l}\text { Grado de incorporación de conocimientos sobre derechos y deberes } \\
\text { del menor en el país de acogida en la programación educativa }\end{array}$} & Hombre & 6 & 3.5000 & .83666 & .34157 \\
\hline & Mujer & 13 & 2.7692 & .83205 & .23077 \\
\hline \multirow{2}{*}{$\begin{array}{l}\text { Incorporación de la participación ciudadana del menor en la } \\
\text { programación educativa }\end{array}$} & Hombre & 6 & 3.3333 & 1.03280 & .42164 \\
\hline & Mujer & 13 & 2.7692 & .83205 & .23077 \\
\hline \multirow[t]{2}{*}{ Se fechan reuniones periódicas entre educador y el menor } & Hombre & 6 & 3.3333 & .81650 & .33333 \\
\hline & Mujer & 13 & 2.4615 & .87706 & .24325 \\
\hline \multirow{2}{*}{$\begin{array}{l}\text { Se proporciona al menor información, material e instrumentos } \\
\text { específicos para su proceso formativo }\end{array}$} & Hombre & 6 & 3.6667 & .81650 & .33333 \\
\hline & Mujer & \begin{tabular}{l|l}
13 \\
\end{tabular} & 3.0769 & .86232 & 23916 \\
\hline
\end{tabular}


Tabla 2: continuación

\begin{tabular}{|c|c|c|c|c|c|}
\hline \multicolumn{2}{|l|}{ Variable 1: Sexo } & & \multirow{2}{*}{\begin{tabular}{|l|} 
Media \\
3.333 \\
\end{tabular}} & \multirow{2}{*}{$\begin{array}{l}\text { Desviación } \\
\text { estándar } \\
.81650\end{array}$} & \multirow{2}{*}{$\begin{array}{l}\text { Media de error } \\
\text { estándar } \\
.33333\end{array}$} \\
\hline Se evalúa el itinerario conjuntamente entre el educador y el menor & Hombre & & & & \\
\hline & Mujer & 13 & 2.6923 & .85485 & .23709 \\
\hline \multirow[t]{2}{*}{ Se discuten los resultados con otros profesionales } & Hombre & 6 & 3.3333 & .81650 & .33333 \\
\hline & Mujer & 13 & 2.7692 & .72501 & .20108 \\
\hline \multirow{2}{*}{$\begin{array}{l}\text { Se realiza una evaluación psicopedagógica previa para atender a la } \\
\text { diversidad }\end{array}$} & Hombre & 6 & 3.5000 & .83666 & .34157 \\
\hline & Mujer & 13 & 3.0000 & .70711 & .19612 \\
\hline \multirow[t]{2}{*}{ Se elaboran informes psicopedagógicos de los menores } & Hombre & 6 & 3.6667 & .81650 & .33333 \\
\hline & Mujer & 13 & 3.0769 & .75955 & .21066 \\
\hline \multirow[t]{2}{*}{ Interviene directamente con menores con dificultades de aprendizaje } & Hombre & 6 & 3.5000 & .83666 & .34157 \\
\hline & Mujer & 13 & 3.0000 & .70711 & .19612 \\
\hline
\end{tabular}

\section{Grado de satisfacción con el puesto}

Todo lo anteriormente expuesto, determina la motivación y el compromiso con la labor que se está realizando, el clima de trabajo y la remuneración recibida. No obstante, en esta labor también influyen las instalaciones y los recursos de los que disponga el educador. En la tabla 3 se muestran los porcentajes de satisfacción que tienen los educadores/as con respecto a estos aspectos. Como se puede apreciar, la remuneración recibida se considera en unos niveles "aceptables" aunque mejorable. El aspecto mejor valorado ha sido el referido al compañerismo entre educadores/as. Por último, se observa una necesidad de mejorar tanto las instalaciones como los recursos disponibles para atender a los menores migrantes.

Tabla 3: Grado de satisfacción con el puesto de educador

\begin{tabular}{|l|l|l|l|l|}
\hline \multirow{2}{*}{$\begin{array}{l}\text { Nivel de } \\
\text { satisfacción }\end{array}$} & \multicolumn{4}{|l|}{$\begin{array}{l}\text { Categorías sobre la satisfacción del puesto de educador } \\
\text { (Porcentaje de respuesta dados por los profesionales) }\end{array}$} \\
\cline { 2 - 5 } & Remuneración & Compañerismo & Instalaciones & Recursos disponibles \\
\hline 0 & $0 \%$ & $0 \%$ & $0 \%$ & $0 \%$ \\
\hline 1 & $0 \%$ & $0 \%$ & $0 \%$ & $0 \%$ \\
\hline 2 & $5,3 \%$ & $0 \%$ & $5,3 \%$ & $0 \%$ \\
\hline 3 & $0 \%$ & $0 \%$ & $0 \%$ & $5,3 \%$ \\
\hline 4 & $15,8 \%$ & $0 \%$ & $5,3 \%$ & $5,3 \%$ \\
\hline 5 & $15,8 \%$ & $10,5 \%$ & $31,6 \%$ & $31,6 \%$ \\
\hline 6 & $21,1 \%$ & $26,3 \%$ & $21,1 \%$ & $26,3 \%$ \\
\hline 7 & $26,3 \%$ & $10,5 \%$ & $15,8 \%$ & $15,8 \%$ \\
\hline 8 & $10,5 \%$ & $10,5 \%$ & $10,5 \%$ & $10,5 \%$ \\
\hline 9 & $0 \%$ & $10,5 \%$ & $0 \%$ & $0 \%$ \\
\hline 10 & $5,3 \%$ & $31,6 \%$ & $10,5 \%$ & $5,3 \%$ \\
\hline
\end{tabular}

\section{DISCUSIÓN FINAL}

La realidad encontrada, nos ha planteado varias cuestiones con el fin de mejorar la intervención realizada en los centros de menores y de acogida. En primer lugar, podemos apreciar una predominancia de las mujeres sobre los hombres en este puesto de trabajo. También, vemos cómo existen diferencias significativas entre los hombres y mujeres en cuanto a la titulación académica, siendo mayor el número de hombres que han cursado estudios de Posgrado y Doctorado. También, vemos como la mayoría de los profesionales indica tener pocos años de experiencia previa en el ámbito de docente con menores destacando aquellos educadoras/es que han trabajado en educación no formal y de adultos.

En segundo lugar, cuando nos fijamos en la formación inicial o durante la carrera profesional los datos arrojan una carencia formativa en aspectos que consideramos esenciales con menores en situación de riesgo. Existe desconocimiento sobre las metodologías de intervención con este tipo de menores así como de la metodología específica de trabajo que utiliza el centro, lo que nos lleva a pensar que quizás no exista una metodología específica establecida para los menores migrantes. Ante esta situación y teniendo en cuenta que, según lo indicado por los educadores/as, en gran medida poseen una situación contractual fija-indefinida pensamos que se debería favorecer este conocimiento y formación dentro de las propias organizaciones. Igualmente, podemos apreciar como muchas de las variables que hacen referencia a la elaboración de objetivos y contenidos relacionados con un desarrollo integral de los menores (aspectos personales, sociales, académicos y profesionales) fue realizada durante la carrera, es decir previa a la incorporación al puesto de trabajo y la mayoría de las personas encuestadas afirman no haberla recibido durante el período de contratación 
En tercer lugar, podemos decir que aparentemente la principal función a desarrollar por los educadores de este tipo de centro se halla en la intervención y desarrollo de actividades. No obstante, no parece llevarse a cabo un seguimiento continuado con los menores a la hora de planificar su itinerario, llegar a acuerdos entre lo que necesita el menor, lo que quiere hacer o la evaluación conjunta de su itinerario, siendo estos aspectos considerados imprescindibles para la inclusión de estos menores a nivel socio comunitario y educativo. También, estos profesionales manifestaron que la coordinación entre el equipo técnico y el grupo de educadores que intervienen con los menores es escasa en muchas ocasiones. Así pues, la planificación de las acciones tutoriales, la elaboración de las distintas programaciones y el trabajo en equipo deja entrever la necesidad de ser mejorado con el fin de aumentar la eficacia en la formación de los menores.

En cuarto lugar, a partir del estudio realizado y de los resultados obtenidos, hemos podido trazar una serie de pautas generales que podrían ser utilizadas para el diseño de un plan formativo específico para educadores que ofrezca una respuesta a las necesidades encontradas anteriormente, y facilite una intervención educativa coordinada que responda a las necesidades reales de los menores en riesgo de exclusión. Este programa buscaría dotar a los educadores de habilidades y competencias relacionadas con el diseño de acciones educativas o metodologías pedagógicas para atender a los menores migrantes.

El diseño de este programa de intervención se sustentaría en el modelo de evaluación propuesto por Stufflebeam y Shinkifield (1987) conocido por las siglas CIPP (contexto, entrada o input, proceso, producto). Estos autores entienden la evaluación como: "Proceso de identificar, obtener y proporcionar información útil y descriptiva acerca del valor y mérito de las metas, la planificación, la realización y el impacto de un objeto determinado, con el fin de servir de guía para la toma de decisiones, solucionar los problemas de responsabilidad y promover la comprensión de los fenómenos implicados" (p.183). Aunque el modelo CIPP responde a un enfoque de evaluación global e integrador (Bausela, 2003), se pueden distinguir dentro del mismo cuatro tipos de evaluaciones (Stufflebeam y Shinkifield, 1987): la evaluación de contexto que consiste en "identificar las virtudes y defectos de algún objeto (...) y proporcionar una guía para su perfeccionamiento" (p.196); la evaluación de entrada se basa en "ayudar a prescribir un programa mediante el cual se efectúen los cambios necesarios" (p.197) para la mejora del contexto; la evaluación del proceso que aporta información continua sobre cómo se está desarrollando un programa y la evaluación del producto que tiene como propósito "valorar, interpretar y juzgar los logros de un programa" (p.201).

Es importante destacar que este modelo de evaluación se relaciona con las distintas fases de planificación de programas. Así pues, Sanz (1990), adaptando el modelo de relación propuesto por Rodgers (1979), propone nueve estadios que hay que seguir en la planificación de un programa y el tipo de evaluación del modelo CIPP con el que se corresponde cada una, los cuales se exponen a continuación: estado 1: análisis de necesidades y estadio 2: desarrollo de metas y objetivos, los cuales se relacionan con la evaluación del contexto; estadio 3: diseño del programa y estadio 4: diseñar un plan de implementación están relacionadas con la evaluación de entrada; estadio 5: plan piloto del programa se relaciona con la evaluación del proceso; estadio 6: evaluar los resultados del plan piloto y estadio 7: adoptar, corregir y abandonar el programa; están relacionados con la evaluación de producto; estadio 8: institucionalizar el programa, se relaciona con la evaluación del proceso; y por último, el estadio 9: evaluar el programa institucionalizado, se relaciona con la evaluación del producto.

El programa de intervención para la mejora de la competencia de los educadores en intervención con menores migrantes, tendría como última finalidad generar "ambientes de aprendizaje" específicos para cada uno de estos menores de forma que se adaptasen a sus necesidades, potencialidades, tiempo y motivaciones personales; siendo los usuarios los que en cualquier momento tuviesen el control de lo que aprenden. Esto debería desarrollarse en coordinación con los profesores de los centros educativos a los que acuden los menores, generándose así un trabajo en red. Este modelo de aprendizaje que se propondría sería híbrido, es decir integraría la modalidad presencial y virtual de aprendizaje. Esto se justifica a través de trabajos como los de Brunner et al, (2017) y Vanderheiden (2006) que ponen de manifiesto el hecho de que las tecnologías promueven la inclusión social, en el sentido de que permiten a las personas aprender, trabajar, viajar, socializar, comprar e interactuar con la comunidad (Manzoor y Vimarlund, 2018). Es decir, "la incorporación de las TIC dentro de las prácticas educativas contribuye a la ampliación de la cultura digital mediante la alfabetización tecnológica que se le proporciona a los educandos" (Martínez - Argüello, et al., 2018), la cual es imprescindible para la inclusión de las personas en la sociedad actual.

El curso se compondría de los siguientes módulos: M1) Habilidades/competencias básicas del educador (25 horas); M2) Características generales de los menores no acompañados, menores en riesgo social y menores infractores (30 horas); M3) Diseño, puesta en práctica y evaluación de acciones educativas con menores no acompañados, menores en situación de riesgo social y menores infractores en el ámbito no formal (40 horas); M4) Diseño, puesta en práctica y evaluación de acciones educativas con menores no acompañados, menores en situación de riesgo social y menores infractores en el ámbito formal (45 horas); M5) Diseño, 
intervención y evaluación de actuaciones de género con menores no acompañadas, en situación de riesgo social y/o menores infractoras en centros formales y no formales (ONGS) (45 horas); M6) Enseñanza aprendizaje de la lengua nativa vehicular del aprendizaje (español) (45 horas); M7) Intervención social con menores no acompañados, menores en situación de riesgo y/o menores infractores (40 horas); M8) Inteligencia emocional en el ámbito educativo no formal (40 horas); M9) Intervención con menores con necesidades educativas específicas de apoyo educativo y/o diagnóstico clínico (40 horas); M10) Aspectos culturales, religiosos y valores de los menores migrantes (30 horas); M11) La mentorización: definición y estrategias básicas para trabajar con menores no acompañados, menores en situación de riesgo y/o menores infractores (40 horas); M12) Coaching educativo y menores no acompañados, menores en situación de riesgo y/o menores infractores (40 horas); y M13) Estrategias de resolución de problemas: aplicación práctica a la intervención con menores no acompañados, menores en situación de riesgo social y/o menores infractores (30 horas).

El programa sería revisado en base a una evaluación combinada: a nivel interno de la adquisición de competencias de los menores (pruebas objetivas, producciones de los menores, simulaciones, observación sistemática); con respecto al propio programa y sus distintos módulos formativos (cuestionarios de satisfacción a los estudiantes, grupo de discusión y triangulación con el resto de formadores que impartan los módulos) y a nivel externo, por agentes de las propias organizaciones en las que desarrollan su trabajo los educadores y el grado de impacto en sus acciones formativas y de intervención a medio largo plazo a través de un seguimiento posterior. Ante todo lo comentando se podría plantear ampliar la muestra y profundizar en las necesidades de los educadores también desde una perspectiva cualitativa. Todo ello, con el objetivo de poder generalizar los resultados a otros contextos y diseñar un programa de intervención para la mejora de las competencias profesionales de los educadores en el trabajo con menores migrantes, el cual sea general para poder adaptarlo a distintos centros de acogida. Así pues, este estudio nos sirve de base y guía para los siguientes pasos a seguir.

\section{CONCLUSIONES}

De acuerdo al trabajo presentado y a los resultados obtenidos, se pueden plantear las siguientes conclusiones:

1.- Los educadores/as de los centros de acogida tiene una serie de necesidades con respecto a la atención socioeducativa de menores extranjeros no acompañados, a las cuales es preciso dar una respuesta para fomentar la inclusión de todas las personas en la sociedad actual.

2.- Las principales necesidades que presentan los educadores/as en la atención del menor extranjero no acompañado son: desconocimiento sobre el proceso específico de intervención socioeducativa, falta de un seguimiento continuado y colaboración con el menor para planificar su itinerario socioeducativo, escasa coordinación con el equipo técnico y el resto de educadores/as del centro, escasa participación en el diseño y planificación de las acciones socioeducativas y de la acción tutorial, falta de recursos e instalaciones para desempeñar de forma adecuada su labor y baja importancia otorgada a los procesos de evaluación y a trabajar con estos menores contenidos interculturales y de participación ciudadana.

3.- Existen algunas diferencias significativas entre los educadores y las educadoras en cuanto a la formación inicial y continua que disponen para atender a los menores migrantes. Esto es importante conocerlo para poder fomentar medidas que fomenten la igualdad de género entre hombres y mujeres en cuanto a su formación siendo este uno de los objetivos de la Agenda 2030 para el Desarrollo Sostenible.

\section{AGRADECIMIENTOS}

Los autores agradecen al Proyecto I+D+l EDU2017-88641-R del Programa Estatal de Fomento de la Investigación Científica y Técnica (MINECO) titulado "Modelos de Aprendizaje Híbridos para la Intervención Educativa con Menores Extranjeros No Acompañados (MENAS). Herramientas Eficaces para la Integración Escolar y Social". Igualmente extienden su reconocimiento al Ministerio de Educación, Cultura y Deporte del Gobierno de España - Formación del Profesorado Universitario - FPU. Referencia: FPU17/03339.

\section{REFERENCIAS}

Bausela, E., Metodología de la investigación evaluativa: modelo CIPP, Revista Complutense de Educación, 14 (2), 361 376 (2003).

Borrego, G., El sistema de protección de menores, Dedica. Revista de Educação e Humanidades, (6), 85-95 (2014).

Brunner, M., Hemsley, B., Togher, L., y Palmer, S., Technology and its role in rehabilitation for people with cognitive communication Disability following a Traumatic Brain Injury (TBI), https://doi.org/10.1080/02699052.2017.1292429, Brain Injury, 31 (8), 1028 - 1043 (2017). 
Cabero, J., e Infante, A., Empleo del método Delphi y su empleo en la investigación en comunicación y educación, https://doi.org/10.21556/edutec.2014.48.187, EDUTEC Revista Electrónica de Investigación Educativa, 48, 1-16 (2014).

Castillo, M., y Gamboa, R. Desafíos de la educación en la sociedad actual, Revista Electrónica Diálogos Educativos, 12 (24), 55-69 (2012).

Comisión Económica para América Latina (CEPAL) y Fondo de las Naciones Unidas para la Infancia (UNICEF), América Latina y el Caribe a 30 años de la aprobación de la Convención sobre los Derechos del Niño, 1- 87, Publicación de las Naciones Unidas, Naciones Unidas, Santiago (2018).

Dziuban, C. D., Howlin, C.P., Moskal, P., Johnson, C., Parker, L. y Campbell, M. Adaptative learning: a stabilizing influence across disciplines and universities, https://doi.org/10.24059/olj.v22i3.1465, Online Learning Journal, 3, 22, 7-39 (2018).

Expósito, J., Romero - Díaz de la Guardia, J.J., Olmos - Gómez, M.C., Chacón - Cuberos, R. y Olmedo - Moreno, E.M. Enhancing skills for employment in the workplace of the future 2020 using the theory of connectivity: shared and adaptive personal learning environments in a spanish context, https://doi.org/10.3390/su11154219, Sustainability, 11 (15), (2019).

Fernández, O., Wellbeing and work: social inclusion of vulnerable groups in northern Spain, https://10.3384/hygiea.14038668.1091353, Hygiea Internationalis, 9 (1), 353-374 (2010).

Jason, H., y Westberg, J., Preparing educators for adaptative education (AE) programs, https://doi.org/10.1080/0142159X.2018.1487049, Journal Medical Teacher, 40, 828-833 (2018).

Jiménez, A.S., Las competencias interculturales en los educadores de menores. Un estudio comparado, https://dx.doi.org/10.4995/reinad.2011.841, Revista sobre la infancia y la adolescencia, 1, 45-56, (2011).

Manzoor, M. y Vimarlund, V., Digital technologies for social inclusion of individuals with disabilities, https://doi.org/10.1007/s12553-018-0239-1, Health and Technology, 8 (5), 377-390 (2018).

Martínez - Argüello, L.D., Hinojo - Lucena, F.J, y Aznar, I., Aplicación de las tecnologías de la información y la comunicación (TIC) en los procesos de enseñanza - aprendizaje por parte de los profesores de química, https://dx.doi.org/10.4067/S0718-07642018000200041, Información Tecnológica, 29 (2), 41-52 (2018).

Martínez - Martínez, A., Olmos - Gómez, M.C., Tomé - Fernández, M., y Omedo - Moreno, E., Analysis of psychometric properties and validation of the personal learning environments questionnaire (PLE) and social integration of unaccompanied foreign minors (MENA), https://doi.org/10.3390/su11102903, Sustainability,11 (10), (2019).

Martínez, P., Pérez, F.J., y Martínez, M., Aplicación de los modelos de gestión de calidad a la tutoría universitaria, https://dx.doi.org/10.5209/RCED.53541, Revista Complutense de Educación, 29 (3), 633-649 (2016).

Martos - García, D., Usabiaga, O. y Valencia - Peris, A., Students' perception on formative and shared assessment: connecting two universities through the blogosphere, https://doi.org/10.7821/naer.2017.1.194, Jounal of New Approaches in Educational Research, 1 (6), 64-70 (2017).

Mikkonen, K., Koskinen, M. Koskinen, C., Koivula, M., Koskimäki, M., Lähteenmäki, M.L., Mäki-Hakola, H., Wallin, O., Sjögren, T., Salminen, L., Sormunen, M., Saaranen, T., Kuivila H.M. y Kääriäinen, M., Qualitative study of social and healthcare educators' perceptions of their competence in education, https://doi.org/10.1111/hsc.12827, Health and Social Care Community, 27, 1555 - 15, 63 (2019).

Ortega, J., Menores migrantes: necesidades educativas y laborales detectadas a través de un estudio cualitativo, https://dx.doi.org/10.25267/Rev_estud_socioeducativos.2019.i7.04, Revista Estudios Socioeducativos (RESED), 7, 55-70 (2019).

Ovalle, D.A., Salazar, O.M, y Duque, N.D., Modelo de recomendación personalizada en cursos virtuales basado en computación ubicua y agentes inteligentes, https://dx.doi.org/10.4067/S0718-07642014000600016, Información Tecnológica, 25 (6), 131-142 (2014).

Pedrazza, M., Berlanda, S., De Cordova, F., y Fraizzoli, M., The changing educators' work environment in contemporary society, https://10.3389/fpsyg.2018.02186, Frontiers in Psychology, 9, 1-12 (2018).

Rodgers, R.F., A student affairs application of the CIPP evaluation model, evaluation in student affairs, America College Personnel Association, 1-273, Michigan, Estados Unidos (1979).

Rodorigo, M., Fernández - Larragueta, S., y Fernández - Sierra, J., La mediación intercultural como herramientas de inclusión: análisis de una experiencia escolar, https://doi.org/10.5209/rced.58885, Revista Complutense de Educación, 3 (30), $747-761$ (2019).

Salmerón - Manzano, E., y Manzano - Agugliaro, F., Unaccompanied minors: worldwide research perspectives, https://doi.org/10.3390/publications7010002, Publications, 7(1), (2018).

Sanz, R., Evaluación de programas en orientación educativa, 1-142, Pirámide, Madrid, España (1990).

Shute, J.W., y Cooper, B.S., Understanding in - school truancy, https://doi.org/10.1177/0031721715575303, Phi Delta Kappan, 96 (6), 65-68 (2015).

Stufflebeam, D., y Shinkfield, A.J., Evaluación sistemática: guía teórica y práctica, 1-381, Paidós, Barcelona, España (1987).

Torres- Martín, C. y Maroto, E. Los aspectos metodológicos de Aldeas Infantiles SOS de Granada, España, Journal for Educators, Teachers and Trainers, 7 (1), 12-26 (2016). 
Tsakitzidis, G., Timmermans, O., Callewaert, N. Truijen, S., Meulemans, H., Van Royen, P. Participant evaluation of an education module on interprofessional collaboration for students in healthcare studies, https://doi.org/10.1186/s12909-0150477-0, BMC Medical Education, 15 (188), 1-8 (2015).

UNICEF Comité Español, Los derechos de los niños y niñas migrantes no acompañados en la frontera sur española, 126, UNICEF Comité Español, Madrid, España (2019).

Vanderheiden, G., Over the horizon: potential impact of emerging trends in information and communication technology on disability policy and practice, National Council on Disability. 1331 F Street NW Suite 850, Washington, DC 20004 (2006). 